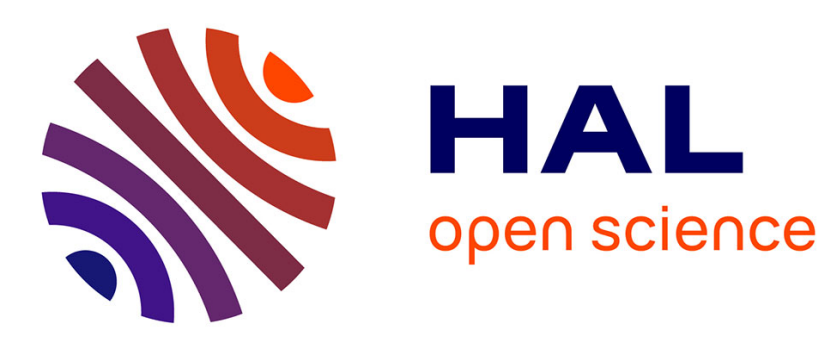

\title{
Threshold of Bénard-Marangoni instability in drying liquid films
}

\author{
Fabien Chauvet, Sam Dehaeck, Pierre Colinet
}

\section{To cite this version:}

Fabien Chauvet, Sam Dehaeck, Pierre Colinet. Threshold of Bénard-Marangoni instability in drying liquid films. EPL - Europhysics Letters, 2012, 99 (3), pp.34001. 10.1209/0295-5075/99/34001 . hal01360014

\section{HAL Id: hal-01360014 https://hal.science/hal-01360014}

Submitted on 5 Sep 2016

HAL is a multi-disciplinary open access archive for the deposit and dissemination of scientific research documents, whether they are published or not. The documents may come from teaching and research institutions in France or abroad, or from public or private research centers.
L'archive ouverte pluridisciplinaire HAL, est destinée au dépôt et à la diffusion de documents scientifiques de niveau recherche, publiés ou non, émanant des établissements d'enseignement et de recherche français ou étrangers, des laboratoires publics ou privés. 


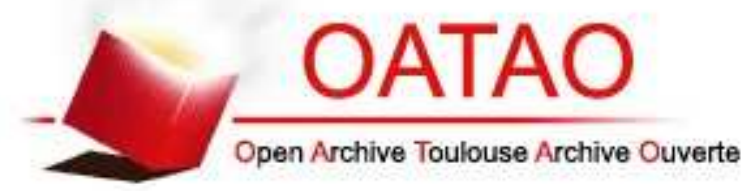

\section{Open Archive TOULOUSE Archive Ouverte (OATAO)}

OATAO is an open access repository that collects the work of Toulouse researchers and makes it freely available over the web where possible.

This is an author-deposited version published in : http://oatao.univ-toulouse.fr/ Eprints ID : 16042

To link to this article : DOI : 10.1209/0295-5075/99/34001

URL : http://dx.doi.org/10.1209/0295-5075/99/34001

To cite this version : Chauvet, Fabien and Dehaeck, Sam and

Colinet, Pierre Threshold of Bénard-Marangoni instability in drying liquid films. (2012) EPL (Europhysics Letters), vol. 99 (n³). pp.

34001-p1-34001-p2. ISSN 0295-5075

Any correspondence concerning this service should be sent to the repository administrator: staff-oatao@ listes-diff.inp-toulouse.fr 


\title{
Threshold of Bénard-Marangoni instability in drying liquid films
}

\author{
F. Chauvet ${ }^{1,2,3(a)}$, S. Dehaeck ${ }^{1}$ and P. Colinet $^{1(b)}$ \\ ${ }^{1}$ Université Libre de Bruxelles, TIPs (Transfers, Interfaces and Processes) \\ C.P. 165/67, 50 Av. F. D. Roosevelt, 1050 Brussels, Belgium, EU \\ ${ }^{2}$ CNRS, LAAS - 7 avenue du colonel Roche, F-31400 Toulouse, France, EU \\ ${ }^{3}$ Univ. de Toulouse, LAAS - F-31400 Toulouse, France, EU
}

PACS 47.20.Dr - Surface-tension-driven instability

PACS 68.03.Fg - Evaporation and condensation of liquids

PACS 47.54.-r - Pattern selection; pattern formation

\begin{abstract}
We here show how evaporation/condensation processes lead to efficient heat spreading along a liquid/gas interface, thereby damping thermal fluctuations and hindering thermocapillary flows. This mechanism acts as an effective thermal conductivity of the gas phase, which is shown to diverge when the latter is made of pure vapor. Our simple (fitting-parameter-free) theory nicely agrees with measurements of critical conditions for Bénard-Marangoni instability in drying liquid films. Heat spreading is also shown to strongly affect wavelength selection in the nonlinear regime. In addition to providing a quantitative framework for analyzing transitions between complex evaporation-driven patterns, this also opens new perspectives for better controlling deposition techniques based on drying.
\end{abstract}

Evaporation and condensation are widespread processes in nature and technology, both at large scales (e.g., water cycle, salt lake drying, ...) and at small scales (e.g., heat exchangers, deposition and coating techniques, ...). In the latter case, such phase change phenomena are generally coupled with so-called Marangoni flows, resulting from surface tension gradients along the interface. For pure liquids evaporating into air, the latter are due to temperature gradients generated by the consumption of latent heat, often resulting in Bénard-type patterns [1,2]. In contrast, when the gas phase contains only vapor (e.g., in boiling applications), such flows are typically absent since the interface is bound to remain close to the saturation temperature (see, e.g., [3], showing the predominance of buoyancy in that case). However, despite the numerous applications, the nature of such interfacial temperature homogenization process remains unclear, and has never been accurately quantified as a function of the vapor content of the gas phase. Intuitively, it can be expected that temperature gets uniformized by the transport of energy (in the form of latent heat) from hot/evaporating to cold/condensing regions along the surface. Our goal here is therefore to assess the efficiency of this "heat spreading"

\footnotetext{
(a) E-mail: fchauvet@gmail.com

(b) E-mail: pcolinet@ulb.ac.be
}

mechanism as a function of fluid properties and ambient conditions.

After having described a simple modeling of this effect in quite general conditions, we analyze its impact on Bénard-Marangoni (BM) patterns in liquid films drying into ambient air, for liquids of different volatilities (the most volatile ones eventually approaching the limiting case of pure vapor). It is well known in that respect that the critical conditions for BM instability somehow depend on thermoconvective processes in the gas phase, generally lumped into an empirically determined heat transfer coefficient [4]. Even though various theories have been proposed to generalize these one-sided approaches and to calculate the (effective) heat transfer coefficients (see, e.g., $[5,6])$, none of them was ever validated by direct comparison with accurate experiments. Actually, the critical Marangoni number $M a_{c}$ for the onset of patterns was experimentally checked only for non-volatile liquids, in which case the value is about $80[4,7]$. In view of the heat spreading mechanism discussed above, one therefore expects a strong damping of the instability, hence much larger values of $M a_{c}$, when volatility increases. In the present work, this is investigated in detail by detecting the transition from the convective to the conductive state occurring when the drying-film thickness decreases below some threshold value. In addition to allowing an accurate 
validation of our new theory, it is worth noting that results presented in this letter could open interesting perspectives to better control techniques using drying films such as polymer coating [8] or nanoparticle deposition [9].

In order to understand and quantify the heat spreading mechanism as a function of the volatility, let us consider a flat interface (at $z=0$, with $z$ pointing to the gas) at temperature $T_{\Sigma}$, where evaporation occurs at a mass flux density $J$ (in $\mathrm{kg} / \mathrm{m}^{2} \mathrm{~s}$ ). Hereafter, a subscript $\Sigma$ will denote a quantity evaluated at the interface, the gas mixture is taken to be perfect, its total pressure $p_{g}$ is supposed to be constant and uniform (small dynamic viscosity), and the inert gas (say, air) is not absorbed into the liquid. This implies (see, e.g., [5])

$$
J=-\left.\frac{D M_{v}}{R T_{\Sigma}} \frac{\partial_{z} p_{v}}{1-\omega}\right|_{z=0},
$$

where $D$ is the vapor-air diffusion coefficient, $M_{v}$ is the molar mass of the vapor, $p_{v}$ is the partial pressure of vapor in the gas phase, $\omega=p_{v} / p_{g}$ its mole fraction, and $R$ is the perfect gas constant. In addition, the energy balance at the interface reads

$$
\left.\frac{\partial T_{l}}{\partial z}\right|_{z=0}=-\frac{J \mathcal{L}}{\lambda_{l}}+\left.\frac{\lambda_{g}}{\lambda_{l}} \frac{\partial T_{g}}{\partial z}\right|_{z=0},
$$

where $T_{l}$ and $T_{g}$ are, respectively, the liquid and gas temperatures, $\mathcal{L}$ is the latent heat of vaporization, while $\lambda_{l}$ and $\lambda_{g}$ are, respectively, the liquid and gas thermal conductivities (with $\lambda_{g} \ll \lambda_{l}$ in general).

We now consider fluctuations (denoted by tilded quantities) around a particular steady (or quasi-steady) state distinguished by a superscript 0 . The determination of this particular state needs not be detailed for the moment, and in principle, the following reasoning applies to both evaporation $\left(J^{0}>0\right)$ and condensation $\left(J^{0}<0\right)$. The interface temperature is written $T_{\Sigma}=T_{\Sigma}^{0}+\widetilde{T}_{\Sigma}$, and assuming local chemical equilibrium at the interface (generally valid except at very small scales $[1,5]$ ), the corresponding fluctuation of vapor partial pressure there reads $\widetilde{p}_{v \Sigma}=p_{\text {sat }}^{\prime}\left(T_{\Sigma}^{0}\right) \widetilde{T}_{\Sigma}$, where $p_{\text {sat }}(T)$ is the coexistence (i.e., Clausius-Clapeyron) curve and a prime denotes its derivative. As fluctuations satisfy $\nabla^{2} \widetilde{p}_{v}=0$ in the limit of a small Péclet number (defined on a typical length scale of the fluctuations, assumed to be much smaller than the typical size $H$ of the gas phase) and in the quasi-static hypothesis, we have $\widetilde{p}_{v \mathbf{q}}=p_{\text {sat }}^{\prime}\left(T_{\Sigma}^{0}\right) \widetilde{T}_{\Sigma \mathbf{q}} \mathrm{e}^{-|\mathbf{q}| z}$, where a subscript $\mathbf{q}$ indicates the Fourier component with wave vector $\mathbf{q}$ (in the horizontal plane). Similarly, one also has $\nabla^{2} \widetilde{T}_{g}=0$, because the Lewis number $L e=D / \kappa_{g}$ is $O(1)$ in the gas ( $\kappa_{g}$ is the gas thermal diffusivity). Hence, assuming $T_{g}=T_{l}\left(=T_{\Sigma}\right)$ at $z=0$, we have $\widetilde{T}_{g \mathbf{q}}=\widetilde{T}_{\Sigma \mathbf{q}} \mathrm{e}^{-|\mathbf{q}| z}$.

Finally, linearizing eq. (1), we can calculate (the Fourier transform of) the phase change rate fluctuation

$$
\widetilde{J}_{\mathbf{q}}=|\mathbf{q}| \frac{D M_{v}}{R T_{\Sigma}^{0}} \frac{p_{\text {sat }} \prime\left(T_{\Sigma}^{0}\right)}{1-\omega_{\Sigma}^{0}} \widetilde{T}_{\Sigma \mathbf{q}}
$$

where fluctuations of the denominator have been neglected (this is rigorously valid for $|\mathbf{q}|^{-1} \ll H$, as will be shown elsewhere). Then, Fourier-transforming the interfacial energy balance (2) and grouping terms, we get

$$
\left.\frac{\partial \widetilde{T}_{l \mathbf{q}}}{\partial z}\right|_{z=0}+\alpha|\mathbf{q}| \widetilde{T}_{\Sigma \mathbf{q}}=0
$$

where

$$
\alpha=\frac{\lambda_{g}}{\lambda_{l}}+\frac{\mathcal{L} D M_{v}}{\lambda_{l} R T_{\Sigma}^{0}} \frac{p_{\mathrm{sat}}^{\prime}\left(T_{\Sigma}^{0}\right)}{1-\omega_{\Sigma}^{0}} .
$$

Equation (4) has the form of a Newton's cooling law for liquid temperature fluctuations, with a heat transfer coefficient depending upon their wave number $|\mathbf{q}|$ (hence, the physical space expression of eq. (4) involves a non-local convolution term). The positive dimensionless number $\alpha$ turns out to be an effective gas-to-liquid ratio of thermal conductivities, accounting for phase-change-induced heat spreading through its second term. In particular, the latter contribution diverges for $\omega_{\Sigma}^{0} \rightarrow 1$, i.e., in the limit of a pure vapor phase, for which eq. (4) implies $\widetilde{T}_{\Sigma \mathbf{q}}=0$, i.e., the interface temperature does not fluctuate and remains equal to the saturation (boiling) temperature.

Now, applying eqs. (4) and (5) to the modeling of evaporation-driven BM convection in a liquid layer of height $e$ much thinner than the gas phase thickness $H$, it turns out that Pearson's theory [4] can be straightforwardly applied (provided we also neglect buoyancy), using an effective Biot number $B i=\alpha k$, where $k=|\mathbf{q}| e$ is the dimensionless wave number. The neutral stability threshold is then given [4] by

$$
M a_{k}=\frac{16 k(k \cosh [k]+\alpha k \sinh [k])(2 k-\sinh [2 k])}{4 k^{3} \cosh [k]+3 \sinh [k]-\sinh [3 k]} .
$$

The critical Marangoni number $M a_{c}(\alpha)$ and the critical wave number $k_{c}(\alpha)$ are then found by minimizing $M a_{k}$ with respect to $k$, and will now be compared to experiments. Note finally that the theory just described appears as a particular case of a more general formulation (not limited to $e \ll H)$ described in [5], and also based on a one-sided reduction of the evaporation-driven BM problem by adiabatic slaving of gas phase fluctuations.

In order to test these predictions, accurate experiments were performed by evaporating thin liquid layers into ambient air $\left(T \approx 24^{\circ} \mathrm{C}\right)$ at rest, until the liquid completely disappears. As explained hereafter, we mostly focus on the moment at which convective patterns disappear in favor of a uniform evaporative state. Volatile liquids used are Hydrofluoroethers, HFE-7000, -7100, -7200 and -7300 from the company $3 \mathrm{M}$, which have similar physical properties except for their saturation pressure $p_{\text {sat }}$ (factor of about 2 between two successive HFEs). HFE-7000 is the most volatile with $p_{\text {sat }}\left(24^{\circ} \mathrm{C}\right)=0.61$ bar and HFE7300 is the less volatile with $p_{\text {sat }}\left(24^{\circ} \mathrm{C}\right)=0.06$ bar. Other thermodynamic and transport properties used hereafter are found in $3 \mathrm{M}$ data sheets (available on $3 \mathrm{M}$ web site). 
Each experimental run is started by pouring a certain amount of $\mathrm{HFE}$ in a cylindrical container to form an approximately $1 \mathrm{~mm}$ thick liquid layer. The container is made of a PVC cylinder glued by silicone to a $10 \mathrm{~mm}$ thick aluminum plate. The height of the cylinder is $1 \mathrm{~cm}$, its diameter is $63.5 \mathrm{~mm}$ and its thickness is $6 \mathrm{~mm}$. In addition to the effect of volatility (dependent on the HFE used), we also vary the evaporation rate independently by changing the "transfer distance" $H$ in the gas. This is accomplished by topping another PVC cylinder (of the same diameter) on the one glued to the plate, wrapping them with a scotch tape in order to avoid any vapor leak. Using additional cylinders of various heights allows to set $H$ to $1 \mathrm{~cm}, 2 \mathrm{~cm}$, $3 \mathrm{~cm}, 4 \mathrm{~cm}$ and $5 \mathrm{~cm}$.

In these conditions, the evaporation process is limited by diffusion of vapor into air and the evaporation rate $E=$ $J^{0} S$ (where $S$ is the container cross-section) remains quasiconstant until the layer is too thin and dewetting begins. The liquid film thickness, $e$, is measured by weighting and is deduced from the measured total mass, $m_{\text {tot }}$, taking into account the mass of the liquid meniscus against the internal cylinder wall, $m_{m e n}$, and the mass of the vapor contained in the gas phase above the liquid, $m_{v}$, such that $e=\left(m_{\text {tot }}-m_{m e n}-m_{v}\right) / \rho_{l} S$, where $\rho_{l}$ is the liquid density. Both these contributions cannot be neglected because the critical layer thicknesses are generally small. More precisely, $m_{m e n}$ is theoretically estimated assuming that the meniscus is in its hydrostatic equilibrium state and that the liquid is perfectly wetting. In turn, $m_{v}$ has been measured experimentally for each $H$ and each HFE (molecular weight between 200 and $350 \mathrm{~g} / \mathrm{mol}$ ) using a suspended thin circular dish filled with liquid and placed very close to the container bottom but without touching the container wall, hence "simulating" the presence of a liquid layer. In the worst case (most volatile liquid HFE-7000 and highest container $H=5 \mathrm{~cm}$ ), we find $m_{v} \simeq$ $0.2 m_{\text {tot }}$ and in the best one (HFE-7300 and $H=1 \mathrm{~cm}$ ), we get $m_{v} \simeq 0.005 m_{t o t}$. The relative uncertainty on the liquid layer thickness measurement is estimated to be lower than $2.5 \%$. The evaporation rate, $E$, is simply computed from the time derivative of the total mass $\left(E=-\mathrm{d} m_{\text {tot }} / \mathrm{d} t\right)$ using a linear fit.

As convection in the pure liquid is necessarily associated with temperature variations, we use a Focal Plane Array Infra-Red (IR) camera-type (Thermosensorik, InSb 640 SM) facing the liquid/gas interface, to follow the time evolution of the whole cellular pattern. IR images and $m_{\text {tot }}(t)$ are recorded at a frequency of $1 \mathrm{~Hz}$ during the drying of the liquid layer. Typically, the observed sequence is similar to the one obtained in [2], i.e., convection appears right after filling and the pattern is strongly time dependent, evolving into more stable hexagonal-like arrangements when $e$ decreases, until the convective state turns into a "conductive" one. The convection cells do not disappear altogether, though. At a certain moment, a straight front separating convective and conductive states starts to propagate along a horizontal direction (at a
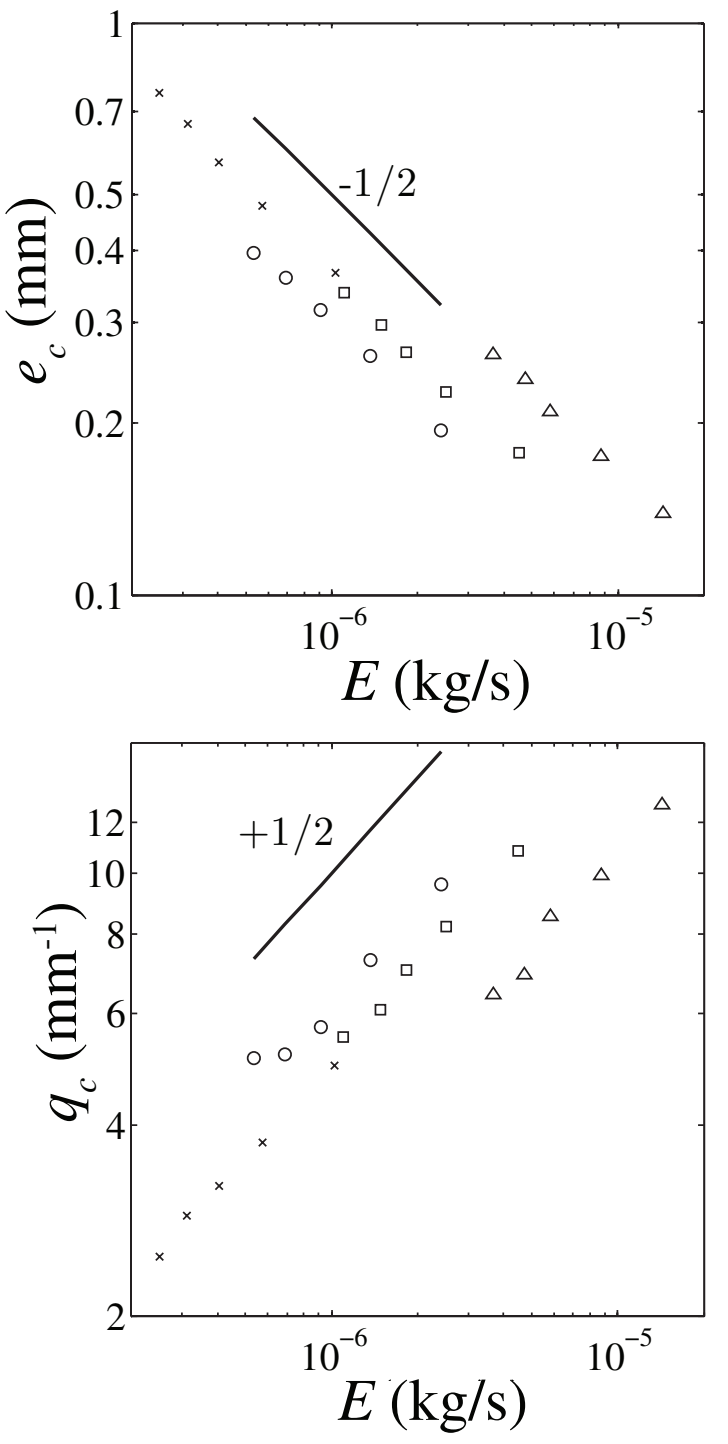

Fig. 1: Measured critical liquid thickness $e_{c}$ (top) and critical wave number $q_{c}$ (bottom) as a function of the evaporation rate $E$, for different liquids (triangles: HFE-7000; squares: HFE7100; circles: HFE-7200; crosses: HFE-7300). Straight lines indicate the theoretical scaling laws (see text).

constant velocity) until convection completely disappears. Performing specific experiments in which the container tilt angle was intentionally slightly varied showed that this front is merely due to a non-absolute horizontality of the layer (the front velocity decreases when increasing the tilt angle and vice versa). We have chosen to define the critical liquid layer thickness, $e_{c}$, by $e_{c}=\left(e_{1}+e_{2}\right) / 2$, where $e_{1}$ is the measured thickness when the front starts to propagate and $e_{2}$ the measured thickness when convection has totally disappeared. For the small tilt angles tested, $e_{c}$ is found to be independent of these small deviations w.r.t. absolute horizontality, hence confirming the suitability of our definition of $e_{c}$. The values obtained are reported in fig. 1 , together with the critical wave number $q_{c}$. Note that 
$e_{c}$ is found to be less that $1 \mathrm{~mm}$ for all cases investigated here, hence justifying our assumption to neglect buoyancydriven (Rayleigh-Bénard) convection.

From the measurement of $e_{c}$ and $E$, we then estimate the critical-temperature difference across the liquid layer, $\Delta T_{c}$, using the thermal balance (2) with a linear temperature profile in the liquid and neglecting heat coming from the gas phase, such that $\Delta T_{c}=E \mathcal{L} e_{c} / \lambda_{l} S$. Then, the critical Marangoni number $M a_{c}$ is calculated as $M a_{c}=$ $-\gamma_{T} \Delta T_{c} e_{c} / \eta_{l} \kappa_{l}$, where $\gamma_{T}$ is the surface tension variation with temperature, $\eta_{l}$ is the liquid dynamic viscosity and $\kappa_{l}$ is its thermal diffusivity. The value of $\gamma_{T}$ has been measured for each HFE by the pendant drop method using the tensiometer Krüss DSA100 with its thermostatted chamber, and a thermocouple placed at the syringe tip end to measure the drop temperature accurately. Surface tension has been measured in the range $15-30{ }^{\circ} \mathrm{C}$, taking special care in order to maintain the drop in a saturated environment (procedure validated by measuring $\gamma_{T}$ of ethanol).

According to our model, the value found for $M a_{c}$ should only depend on the fluids used (liquid and gas), through the value of $\alpha$ given by eq. (5), and characterizing the damping of thermal fluctuations at the interface. Note that in eq. (5), the mole fraction at the interface in the reference state is given by $\omega_{\Sigma}^{0}=p_{\text {sat }}\left(T_{\Sigma}^{0}\right) / p_{g}$, where $p_{g}$ is the ambient pressure. Then, even though $\alpha$ in principle depends on the temperature $T_{\Sigma}^{0}$ in the reference state, hence on the evaporation rate $E, T_{\Sigma}^{0}$ can in general be safely taken equal to the ambient temperature, at least for the diffusionlimited regimes considered here (small temperature drop across the liquid film). Therefore, as $\Delta T_{c} \sim E e_{c}$ for a given liquid and lateral size of the container (see above), $M a_{c} \sim E e_{c}^{2}$ should be independent of the evaporation rate $E$, leading to the scaling $e_{c} \sim E^{-1 / 2}$. Apart for some small variations studied hereafter, the size of convection cells at threshold should be roughly proportional to the depth $e_{c}$. Hence, the critical wave number $q_{c} \sim e_{c}^{-1} \sim E^{1 / 2}$. Both these scalings indeed match experimental measurements, as shown in fig. 1 .

Now, in order to fully validate our Pearson-like theory, $\alpha$ is directly computed from eq. (5) using our own measured values of $D$ for each HFE, obtained by Stefan's tube method [10]. The obtained values of $M a_{c}$ for all the HFEs and for all the container heights $H$ are plotted as a function of $\alpha$ in fig. 2, and compared to the theoretical law $M a_{c}(\alpha)$ obtained from eq. (6), and independent of $E$ as already mentioned.

The inset of fig. 2 also shows the dimensionless critical wave number of the pattern, $k_{c}=q_{c} e_{c}$, clearly independent of $E$ as well, and slightly decreasing with $\alpha$ in agreement with the theoretical curve $k_{c}(\alpha)$. Note that the critical wave number $q_{c}$ (as also plotted in fig. 1) and the wave number $q$ (during the convection regime) are measured as the mean position of the fundamental peak in azimuthally averaged FFT spectra of IR images (see fig. 3). Error bars on $k_{c}$ in fig. 2 correspond to the width at middle

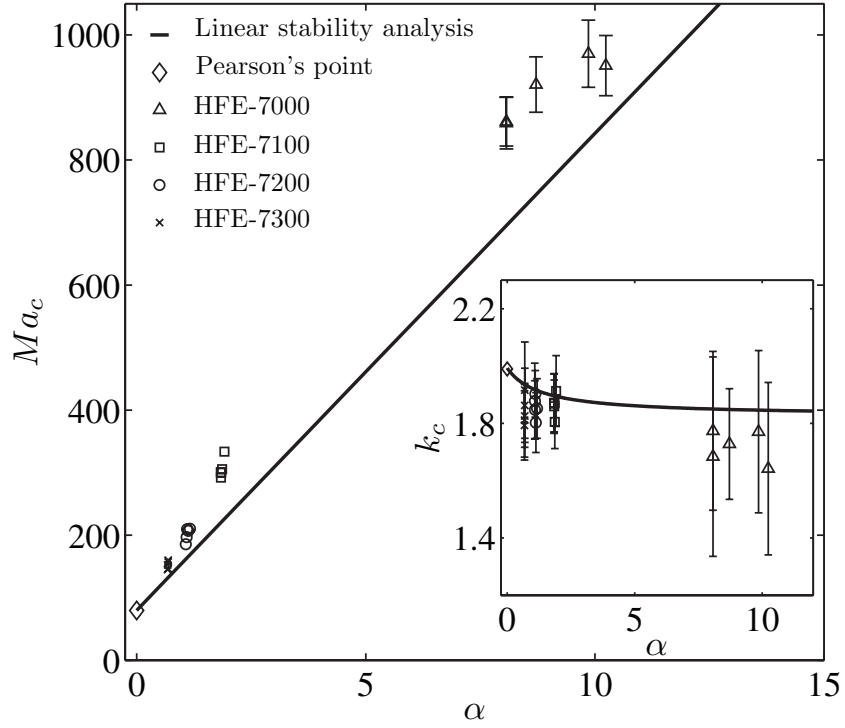

Fig. 2: Measured critical Marangoni numbers (symbols) as a function of $\alpha$, for the four different liquids and the five container heights. Inset: corresponding measured critical wave numbers. Theoretical laws are shown as plain curves.

height of the fundamental peak, hence they are indicative of the level of disorder in the pattern (higher at high volatility).

Figure 2 demonstrates the strong stabilizing effect of the liquid volatility, as well as a quite satisfactory agreement with our simple one-sided theory (given typical uncertainties remaining on some fluid properties and the absence of fitting parameters). This also confirms the effectiveness of the heat spreading mechanism described by eqs. (4) and (5). Note, in addition, that this nice agreement actually validates a number of assumptions made in such type of one-sided models (see also [5]), such as small gas viscous stresses, low Péclet numbers in both phases, quasi-steadiness of the approach despite the continuously decreasing liquid depth, undeformable interface, absence of temperature discontinuity,.... In addition, we emphasize that the simplest form of the theory presented here relies on the additional assumption of a large gas thickness $H$ compared to the liquid depth $e$. As the length scale of convective fluctuations typically scales with $e$, their penetration depth in the gas is of the same order, which in fact allows neglecting the effects of gas density variations and diffusion-induced convection (even though both these effects do affect the homogeneous evaporation state, hence $J^{0}$, when $\omega_{\Sigma}^{0}$ is not small). This will be detailed elsewhere.

To summarize, the results obtained so far evidence an original and somehow unexpected effect of the liquid volatility, which appears to act in two opposite ways. On the one hand, evaporation leads to consumption of latent heat, hence globally cooling the interface and giving rise to thermocapillary convection. On the other hand, 


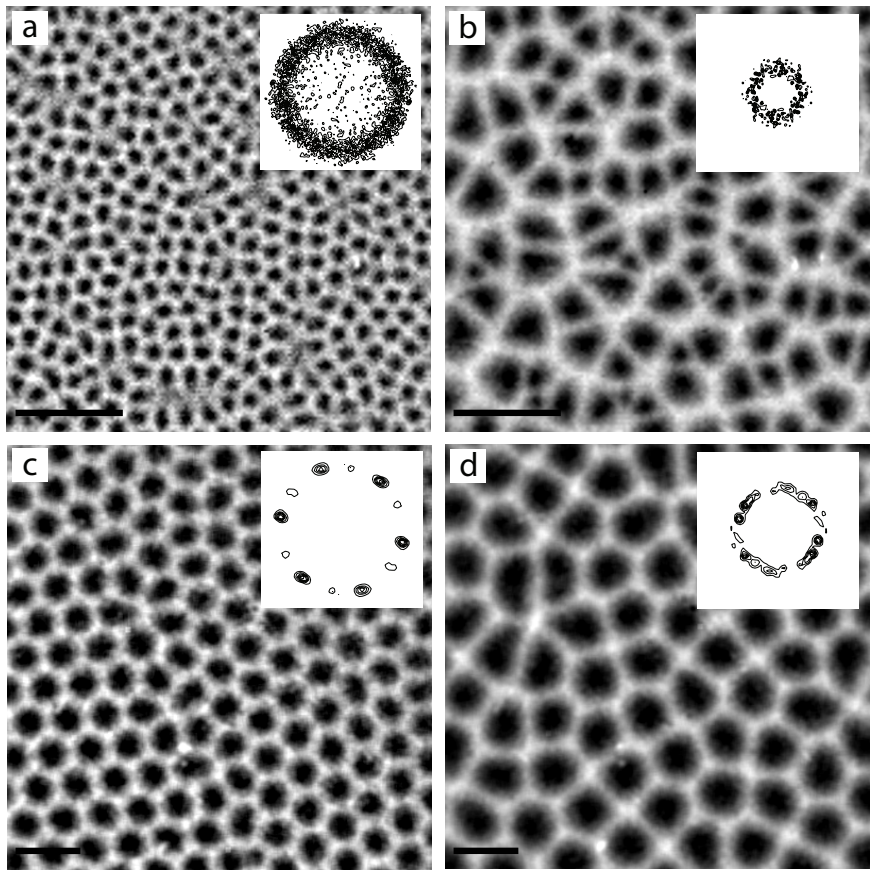

Fig. 3: Typical evaporation-driven $\mathrm{BM}$ patterns $(H=3 \mathrm{~cm})$ : (a) HFE-7000, $\epsilon=0.2$; (b) HFE-7000, $\epsilon=2$; (c) HFE-7300 $\epsilon=0.2$; (d) HFE-7300, $\epsilon=2$. White bars are $4 \mathrm{~mm}$ long. Topright insets: contour lines of power (Fourier) spectrum.

volatility also allows heat to spread from locally hot to cold regions of the interface by diffusion of vapor in the gas. Interestingly, this mechanism appears to act as if the gas phase had a large thermal conductivity. For the case investigated here, where the gas phase is much thicker than the liquid (note that this actually corresponds to most practical applications using drying films), $M a_{c}$ is found to depend mostly on the liquid volatility (a material property, only dependent upon ambient temperature and pressure), and is in particular independent on the particular value of the evaporation rate (controlled by varying the gas phase thickness). The dimensions of convective cells at threshold (i.e., $q_{c}^{-1}$ laterally and $e_{c}$ vertically) in turn significantly depend on the evaporation rate (see fig. 1). This result is potentially of great interest for the rapidly developing coating/deposition techniques based on film drying, which allow self-organized large-scale assembly of small-scale (sub-millimetric) deposits [9]. Even if additional transport phenomena might be involved in these situations (solute diffusion, solutal Marangoni effect,...), one can expect that controlling the solvent evaporation rate externally should allow modifying the lateral size of deposits. Further analysis would be needed, however, as assessing this possibility would require determining at which moment particles settle down or accumulate during drying of the film.

As a conclusion, let us briefly explore nonlinear regimes of evaporative BM convection. Note, however, in this respect that even though buoyancy-driven convection

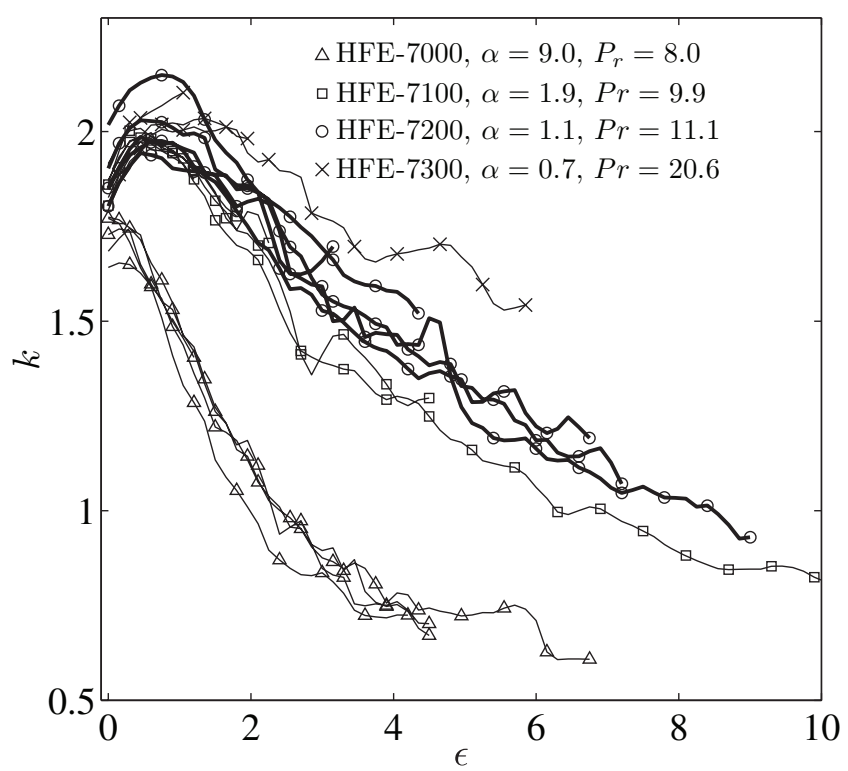

Fig. 4: Measured dimensionless wave number $k$ vs. supercriticality $\epsilon=\left(M a-M a_{c}\right) / M a_{c}$. For each liquid, evolutions corresponding to all five container heights are represented. $\mathrm{Pr}$ is the Prandtl number of the liquids.

turned out to be negligible near the threshold (see above), this might not be the case at larger liquid depths (nor at very large values of $\alpha$, not considered here, for which heat spreading efficiently homogenizes the interfacial temperature, hence strongly mitigating BM convection in favor of the Rayleigh-Bénard instability [3]). Figure 3 shows that cellular patterns become more regular when the supercriticality $\epsilon=\left(M a-M a_{c}\right) / M a_{c}$ decreases (i.e., when time goes on), and that at the same value of $\epsilon$, patterns are more disordered for more volatile liquids. This is also confirmed by the corresponding Fourier spectra, which clearly have larger width and less orientational order for the most volatile HFE-7000 (figs. 3(a) and (b)) than for the less volatile HFE-7300 (figs. 3(c) and (d)).

From these Fourier spectra, the averaged wave numbers $k=q e$ can also be extracted, and are depicted in fig. 4 . We first note that the shape of $k(\epsilon)$ curves (including the nonmonotonic behavior at low $\alpha$ ) is in nice qualitative agreement with direct simulations of [11], which, however, rely on a constant Biot number instead of eq. (4). Interestingly, fig. 4 also shows that the measured wave number evolutions are rather independent of the container height (hence of the evaporation rate $E$ ), while they do depend on the liquid used. This clearly has to do with the fact that the time scale for liquid depth variation, $\tau_{e} \sim e /|\dot{e}|$, is always much larger than the thermal time scale $\tau_{t h} \sim e^{2} / \kappa_{l}$, allowing to expect a quasi-steady regime to be reached at each instant. However, the fact that $\tau_{e}$ turns out to be much smaller than the lateral diffusion time $\tau_{L} \sim L^{2} / \kappa_{l}$ (where $L$ is the container size) points to a rather fast mechanism of wavelength selection, which might be due, at least far from threshold where the pattern is large scale $\left(k \ll k_{c}\right)$, 
to the anomalous dissipation mechanism described by eq. (4).

This remains to be studied, however, along with the quite unexplored scenarios of transition to (or from) "interfacial turbulence" (see also [11]), for which the one-sided model we propose in this letter should be appropriate in a quantitative sense. Note finally that although validated here using a Bénard set-up, the phasechange-induced homogenization mechanism described by eq. (4) is expected to be generic for other geometries as well (e.g., drops, bubbles, ...), at least for sufficiently short-scale interfacial temperature fluctuations.

The authors are grateful to A. A. NePOMNYASHCHY, A. Oron, A. Rednikov, M. Bestehorn and R. NARAYANAN for interesting discussions, and acknowledge financial support of ESA and BELSPO PRODEX projects, EU - FP7 MULTIFLOW Initial Training Network, ULB - BRIC, and Fonds de la Recherche Scientifique FNRS.

\section{REFERENCES}

[1] Colinet P., Legros J.-C. and Velarde M. G., Nonlinear Dynamics of Surface-Tension-Driven Instabilities (Wiley-VCH, Berlin) 2001.

[2] Mancini H. and Maza D., Europhys. Lett., 66 (2004) 812 .

[3] Kimball J. T., Hermanson J. C. and Allen J. S., Phys. Fluids, 24 (2012) 052102.

[4] Pearson J. R. A., J. Fluid Mech., 4 (1958) 489.

[5] Haut B. and Colinet P., J. Colloid Interface Sci., 285 (2005) 296.

[6] Sultan E., Boudaoud A. and BenAmar M., J. Fluid Mech., 543 (2005) 183.

[7] Schatz M. F., VanHook S., McCormick W., Swift J. and Swinney H., Phys. Rev. Lett., 75 (1995) 1938.

[8] Bassou N. and Rharbi Y., Langmuir, 25 (2009) 624.

[9] Maillard M., Motte L., Ngo A. T. and Pileni M. P., J. Phys. Chem. B, 104 (2000) 11871.

[10] Abraham A. J. and Bean C. P., Am. J. Phys., 57 (1989) 325330.

[11] Merkt D. and Bestehorn M., Physica D, 185 (2003) 196. 